\title{
Conciencia ambiental y sostenibilización curricular, dos herramientas en el camino hacia la sostenibilidad de la Universidad de Córdoba
}

\author{
Francisco Villamandos de la Torre \\ Aula de Sostenibilidad de la Universidad de Córdoba. Córdoba. España. \\ bv1vitof@uco.es \\ ORCID: https://orcid.org/0000-0002-3627-4738
}

Antonio Gomera Martínez

Servicio de Protección Ambiental de la Universidad de Córdoba. Córdoba. España. agomera@uco.es

ORCID: https://orcid.org/0000-0003-0603-3017

\author{
Miguel Antúnez López \\ Aula de Sostenibilidad de la Universidad de Córdoba. Córdoba. España. \\ m.antunez@uco.es \\ ORCID:https://orcid.org/0000-0001-5358-6947
}

[Recibido: 21 Enero 2019. Revisado: 22 Enero 2019. Aceptado: 23 Enero 2019]

\begin{abstract}
Resumen: El equipo de la Universidad de Córdoba autor de este trabajo lleva ya más de treinta años autodefiniéndose. Partiendo de un planteamiento de la educación ambiental más naturalista y centrado en los problemas de la conservación de la naturaleza, se ha ido derivando a planteamientos muchos más cercanos a la idea de sostenibilidad y sus objetivos definidos para el horizonte 2030. El presente artículo se dirige a realizar una revisión del compendio de trabajos e investigaciones desarrolladas, con un doble objetivo. El primero es el de presentarnos a la comunidad científica destinataria de esta nueva publicación, como grupo de trabajo universitario en Educación Ambiental (integrado en el grupo de investigación SEJ049 Evaluación educativa e innovación). Por otro, el de divulgar los hallazgos obtenidos y reflexionar sobre su utilidad. El título del artículo hace referencia a las dos grandes líneas de trabajo en las que nos estamos desenvolviendo: el estudio de la conciencia ambiental y de los procesos de sostenibilización curricular, con la formación del profesorado como principal catalizador.
\end{abstract}

Palabras clave: Educación Ambiental, Educación para la Sostenibilidad, Formación del Profesorado, Sostenibilización Curricular, Conciencia Ambiental, Universidad.

Environmental awareness and curriculum sustainability, two tools on the road towards the sustainability of the University of Córdoba

Abstract: The team of the University of Córdoba author of this work has been self-defining for more than thirty years. Starting from a more naturalistic approach to environmental education focused on the problems of nature conservation, it has been evolving to approaches much closer to the idea of sustainability and its objectives defined for the 2030 horizon. This article aims to carry out a review of the compendium of works and research developed, with a double objective. The first is to present ourselves to the scientific community that is the receiver of this new publication, as a university work group on Environmental Education (integrated in the research group SEJ049 Educational evaluation and innovation). Secondly, to disseminate the findings of our research and reflect on its usefulness. The title of the article refers to the two main lines we are working in: the study of environmental awareness and curriculum sustainability processes, with teacher training as its main catalyst.

Keywords: Environmental Education, Education for Sustainability, Teacher Training, Curriculum Sustainability, Environmental Awareness, University 
Para citar este artículo: Villamandos, F., Gomera, A. y Antúnez, M. (2019) Conciencia ambiental y sostenibilización curricular, dos herramientas en el camino hacia la sostenibilidad de la Universidad de Córdoba. Revista de Educación Ambiental y Sostenibilidad 1(1) 1301. doi: 10.25267/Rev_educ_ambient_sostenibilidad.2019.v1.i1.1301

\section{Presentación}

La Educación Ambiental, como cuerpo doctrinal, es aún un proceso en fase de concreción. Son muchas las corrientes (Sauvé, 2004), tipologías y escenarios (Junta de Andalucía, 2006) que coexisten sin constituir aún un entramado epistemológico claro y definido. Sin embargo, es perentoria la necesidad de dar respuestas profesionales y bien fundamentadas a la demanda social urgente sobre el deterioro y la crisis ambiental actual.

El equipo de la Universidad de Córdoba, autor de este trabajo, lleva ya más de treinta años autodefiniéndose. Partiendo de un planteamiento de la educación ambiental más naturalista y centrado en los problemas de la conservación de la naturaleza, se ha ido derivando a planteamientos muchos más cercanos a la idea de sostenibilidad y sus objetivos definidos para el horizonte 2030.

Este proceso no ha sido lineal ni fácil ni unidireccional, pero sí que ha sido guiado en toda su trayectoria por el objetivo de dotar a este cuerpo doctrinal del conocimiento y de la experiencia práctica que fundamentan el complejo rol que las universidades han de jugar en el contexto actual. El presente artículo se dirige a realizar una revisión del compendio de trabajos e investigaciones realizadas con un doble objetivo. El primero es el de presentarnos a la comunidad científica destinataria de esta nueva publicación, como grupo de trabajo universitario en Educación Ambiental (integrado en el grupo de investigación SEJ049 Evaluación educativa e innovación). Por otro, el de divulgar los hallazgos obtenidos y reflexionar sobre su utilidad. El título del artículo hace referencia a las dos grandes líneas de trabajo en las que nos estamos desenvolviendo.

\section{El escenario}

La Universidad es muy relevante a la hora de proporcionar respuestas a los problemas y retos de la sociedad actual y futura (Gutiérrez, Benayas y Calvo, 2006). Además, constituye un agente dinamizador del cambio para la sostenibilidad de especial relevancia, pues forma futuros profesionales que, al ejercer, influirán directa o indirectamente en su entorno (Martínez-Agut, Aznar, Ull y Piñero, 2007) a través de sus conocimientos, valores y actitudes (Gomera, Villamandos y Vaquero, 2012).

Podríamos decir que hemos pasado en las últimas décadas de intentar ambientalizar los campus (Benayas, Alba y Sánchez, 2002) a querer sostenibilizar las universidades (Benayas y Alba, 2011). Es decir, el objetivo ha evolucionado desde establecer medidas para gestionar de forma responsable con el medio ambiente las actividades con mayor impacto a, en la actualidad, desarrollar estrategias que permitan avanzar hacia universidades más sostenibles sistémicamente, en la que la gestión de los aspectos ambientales sea una de las partes a considerar (Antúnez, 2017).

En este sentido, en la Universidad de Córdoba (en adelante, UCO) se ha desarrollado un proceso equivalente. Desde 2001 se cuenta con el Servicio de Protección Ambiental (SEPA), órgano encargado de la gestión ambiental en dicha universidad. 
Sus primeros objetivos se centraron en la correcta gestión de los residuos peligrosos. Poco a poco se fueron introduciendo líneas de acción en otros ámbitos, todos ellos tendentes a la mejora en la gestión ambiental con la perspectiva puesta en la ambientalización de los campus de la UCO.

Por otro lado, el Aula de Sostenibilidad (AS) de la UCO, integrada en el SEPA, nació en enero del 2008 para dar respuesta al creciente interés que el binomio de la Educación Ambiental y la Sostenibilidad estaba adquiriendo en distintos escenarios, entre ellos, la Universidad (Aula de Sostenibilidad, 2017). La coordinación de los dos órganos fortalece la relación entre la gestión ambiental y la educación ambiental, y ha dado lugar, entre otros avances, a interesantes procesos participativos para la mejora ambiental de la institución, como el descrito en el trabajo de León-Fernández, Gomera, Antúnez, Martínez-Escrich, Villamandos y Vaquero (2017). Pero, además, comienza con ello a vislumbrarse una evolución hacia propuestas más integrales en el marco de la sostenibilidad y en las que se afianza la idea de la universidad como agente fundamental en el necesario cambio social. Se trata de fortalecer el papel de la universidad como creadora de profesionales, que van a mostrar un desempeño más o menos sostenible dependiendo, principalmente y entre otras variables, de la educación recibida. La gestión ambiental se convierte así en una pata fundamental de un proceso mucho más ambicioso, el de transformación de la UCO en una universidad sostenible.

Personas pertenecientes al equipo de investigación autor de este trabajo, llevan trabajando desde hace más de 30 años en el campo de la Educación Ambiental, entendiéndola como una herramienta práctica y determinante para la resolución de los problemas ambientales, asumiendo que éstos han de ser interpretados aceptando su complejidad. En esta línea, se participó en el desarrollo y posterior publicación tanto del Libro Blanco de la Educación Ambiental en España (Ministerio de Medio Ambiente, 1999) como en 2003, de la Estrategia Andaluza de Educación Ambiental. Ambos constituyen un marco de referencia fundamental para movilizar recursos y poner en práctica políticas transversales en todos los escenarios de actuación. De hecho y en concreto, fue a raíz de la publicación de la Estrategia Andaluza mencionada cuando, desde Córdoba, se iniciaron los contactos con el resto de las universidades andaluzas para la concreción de una formación de educadores ambientales de nivel universitario y en forma de título oficial. Fue ya en el curso 2009-10 cuando arrancó el Máster Interuniversitario en Educación Ambiental como un título de posgrado oficial al amparo del Espacio Europeo de Educación Superior. Paralela y coordinadamente, en el seno de la propia UCO, este equipo comenzó a dinamizar y promover diversas estrategias de cara a acercar a la institución a modelos más sostenibles, empleando la educación ambiental como principal instrumento. En un principio de manera intuitiva y posteriormente más planificada y coordinada, aprovechando la excelente oportunidad que ofrecía el hecho de pertenecer a los órganos institucionales de gestión y educación ambiental, como son los mencionados SEPA y AS. Esta integración entre gestión, investigación y educación ambiental ha propiciado en los últimos años numerosos y contrastables avances en el proceso de ambientalización de la UCO.

Estas estrategias han ido ligadas a diversas líneas de investigación abiertas a lo largo de los años, focalizadas sobre los colectivos tanto de estudiantes como de profesorado. La primera de ellas se ha centrado en el estudio de la conciencia 
ambiental del alumnado universitario como base para el establecimiento de acciones de educación ambiental. Otra línea de actuación se enmarca en el análisis de los procesos de sostenibilización curricular, poniendo especial énfasis en la formación del profesorado como catalizador de los avances en dichos procesos. Ambos ejes de trabajo han dado lugar a numerosas contribuciones científicas de ámbito nacional e internacional, que se exponen a continuación.

\section{Sobre formación de la conciencia ambiental (CA)}

El acercamiento teórico a esta importante variable del comportamiento proambiental se realiza desde modelos teóricos que parten de asunciones como la necesidad de cambiar el Paradigma Social Dominante a un Nuevo Paradigma Ecológico (Amérigo, Aragonés, Sevillano y Cortés, 2005). Partimos de la definición de Conciencia Ambiental de Febles (2004) y de los modelos multifactoriales de la misma expuestos ya por Chuliá (1995), para diseñar un instrumento para describir las distintas dimensiones en torno a las cuales se define este concepto en el ámbito universitario, compartiendo en ciertos aspectos estructura similar con los utilizados en algunos estudios sobre concienciación ambiental, como los Barómetros CIS o el Ecobarómetro andaluz (Valencia, Arias y Vázquez, 2010; Jiménez y Lafuente, 2006) o la escala del Nuevo Paradigma Ecológico (NEP) (Dunlap y Van Liere, 1978; Dunlap, Van Liere, Merting y Jones 2000; Vozmediano y Sanjuan, 2005).

\section{La primera investigación sobre conciencia ambiental}

La primera investigación en este campo la constituyó una tesis doctoral (Gomera, 2011) cuyos resultados dieron origen a dos publicaciones que recogían sus principales hallazgos. Por una parte, la medición y categorización de la CA del alumnado universitario, analizando la contribución de la universidad a su fortalecimiento (Gomera, Villamandos y Vaquero, 2012) y, por otra, la construcción de indicadores de creencias ambientales a partir de la escala NEP (Gomera, Villamandos y Vaquero, 2013).

Se diseñó el cuestionario Conciencia ambiental en los centros universitarios para el estudio de la CA del alumnado, a partir de las cuatro dimensiones que conforman este concepto: cognitiva (información, conocimiento), afectiva (creencias, valores, percepciones), conativa (actitudes, sentimientos de responsabilidad) y activa (comportamientos individuales y colectivos). Tras una primera redacción, el cuestionario se sometió a validación mediante juicio de expertos y prueba piloto.

La modalidad de cuestionario seleccionada fue autoadministrado de tipo mixto, contemplando diferentes tipos de respuesta. Posteriormente se homogenizaron los criterios de codificación generando valoraciones escalares, con el fin de poder trabajar conjuntamente con todos los ítems o indicadores construidos a partir de ellos.

Como resultados más destacables se encontró, por un lado, que la población estudiantil podía categorizarse en tres tipos(Gomera, Villamandos y Vaquero, 2012):

CA mayor: describe una CA alta que, con matices, muestra un relevante nivel de conocimiento, información, valores y creencias, actitudes y comportamientos ambientales. 
CA menor: corresponde a un perfil de estudiantes con un nivel más bajo o difuso de $\mathrm{CA}$, en el que, aun partiendo de un aceptable nivel cognitivo, no se perciben valores y creencias proambientales ni un sentimiento de responsabilidad personal, que derivan en una actitud pasiva o negativa frente a la participación, así como en patrones de conducta no respetuosos con el medio ambiente, salvo aquellos de bajo esfuerzo o coste.

Sujetos no condicionados por la CA: Más allá de estos dos grupos, encontramos un tercer sector, correspondiente a los sujetos cuyos comportamientos más o menos respetuosos con el medio ambiente no están condicionados por la medida de CA definida en el presente estudio. No es, por tanto, un grupo cuya conducta ambiental sea negativa, sino que no depende de la CA caracterizada ni es consecuencia de la adquisición de competencias para la acción. Como señala Breiting (1997), se trata de comportamientos que pueden componerse del mismo tipo de actos que las acciones, pero no son intencionados al no ser perseguidos como objetivos.

\section{Incidencia de la universidad en la CA del alumnado}

Pero, además, encontramos un hallazgo que para nosotros fue sorprendente: la escasa incidencia del fortalecimiento de la CA a lo largo de los años de formación universitaria.

Recopilando y analizando con detalle el conjunto de resultados obtenidos, se aprecia que en la población de referencia el nivel de CA guarda relación con las variables de carácter académico con el que el alumnado accede a la universidad (titulación, centro, área de conocimiento, campus), pero no con las relacionadas con la evolución del alumnado durante su etapa universitaria (curso o ciclo). Este hecho puede dar lugar a la interpretación de que el nivel de CA que posee y va adquiriendo el alumnado parece no evolucionar por causas relacionadas con la educación universitaria. Este hallazgo no esperado deriva en que la Universidad parece no aportar ganancia significativa de CA durante el paso del alumno por ella, de lo que se infiere que aún no ha incorporado procesos efectivos para la introducción del medio ambiente y la sostenibilidad en el curriculum, tanto en las enseñanzas formales como las no formales.

Ello implica la formulación de dos nuevos interrogantes. Por una parte, si esto es así, cómo y cuándo se forma esa CA antes de entrar en la universidad. Por otro lado, si llegamos a ser capaces de identificar la respuesta a la primera pregunta, deberíamos también identificar cuáles son las variables que han incidido antes del periodo universitario en nuestros estudiantes para comprobar si éstas están o no presentes en la vida universitaria.

En un estudio posterior (Gomera, Villamandos y Vaquero, 2013) presentamos una propuesta metodológica que posibilita potenciar la utilidad y aplicabilidad de la Escala NEP, por medio de la construcción de indicadores específicos de creencias ambientales a partir de sus ítems, que pueden ser incorporados dentro de sistemas generales de medición de la conciencia ambiental, contribuyendo además a profundizar en el entendimiento y fortalecimiento de este concepto.

Quedaba clara la utilidad del instrumento desarrollado y nos daba pie a seguir usándolo en investigaciones posteriores, las cuales se han desarrollado principalmente a través de diversos Trabajos de Fin del Máster Interuniversitario de 
Educación Ambiental mencionado, en cuya dirección o colaboración han participando autores del presente trabajo.

\section{Buscando el origen de la formación de la CA de nuestro alumnado: los centros preuniversitarios}

Así, parecía evidente que la CA del alumnado universitario viene determinada previamente a su ingreso en la universidad y no se altera durante la misma. Además, los datos también nos revelan que, es esa CA previa, la que podría estar determinando le titulación que eligen. Por ello se emprendió un estudio en el que se abordaba el Análisis y medición de la CA en el alumnado de primer curso de la UCO (Gil, 2016). En él se aplicó una versión modificada del cuestionario utilizado en los estudios anteriores con el fin de ser aplicado al alumnado de nuevo ingreso durante su primera semana de estancia en la universidad. Con este trabajo se intenta indagar tanto el nivel de CA del alumnado antes de ser influenciado por la universidad, como buscar correlaciones con los centros de secundaria de procedencia.

Aparte de otros hallazgos de interés, este estudio arrojó que, efectivamente, existe una marcada correlación entre el nivel de CA del alumnado que ingresa a la universidad con el centro del que proceden, poniéndonos ante la evidencia de que la conformación de la CA puede estar ya muy condicionada en la edad adolescente. En cualquier caso, las diferencias entre unos centros de enseñanza y otros podrían ser determinantes en el nivel de esa CA, por lo que sería interesante analizar esta cuestión en mayor profundidad para conocer cuáles son los factores determinantes en el centro de procedencia a fin de elaborar propuestas que mejoren las estrategias desarrolladas en estos.

\section{El programa Ecoescuela como generador de una mayor CA}

Con todo esto como antecedente, decidimos abordar algunos estudios posteriores como: Incidencia del Programa Ecoescuela en la definición de la conciencia ambiental del alumnado. Un estudio comparado (Morales, 2016) sobre la incidencia del programa Ecoescuela en esta determinación.

El objetivo general de esta investigación fue comparar la tipificación de la CA entre dos poblaciones de alumnado de dos centros de Educación Secundaria Obligatoria que comparten un mismo ideario, pero que se diferencian en que en uno de ellos se ha desarrollado el programa Ecoescuela (cuyo objetivo es impulsar la Educación Ambiental en la vida de los centros escolares, implicando a todo su entorno; así como crear una red de centros educativos donde se favorezcan los intercambios y la cooperación (Junta de Andalucía, 2015)) y en el otro no.

Los principales hallazgos orientaban a que el alumnado del centro Ecoescuela parece considerarse más informado por el centro educativo en relación a cuestiones ambientales, por lo que es posible que el proyecto Ecoescuela sea una variable que afecta al centro $y$, por lo tanto, incide en la cantidad de información/formación proporcionada. Además, el centro Ecoescuela es considerado por el alumnado con una mejor situación y evolución medioambiental.

Este estudio abría una serie de líneas de investigación relacionada con la búsqueda de otras variables de incidencia en la percepción de la CA que permitan una medición concreta de la misma (entorno familiar, entorno social...) y darle más visibilidad al 
programa Ecoescuela para fomentar en los centros educativos la realidad ambiental existente y actuar en consecuencia.

\section{Otras variables que, a priori, podrían conformar la CA}

En un análisis posterior, intentamos comprobar la incidencia que el entorno familiar podría también tener en la formación de la CA de chicos de 12 a 16 años: Análisis comparado de la conciencia ambiental en educación secundaria: la visión del alumnado y sus familias (Águila, 2017).

Tras actualizar y adaptar el instrumento de medición de CA y analizar los datos recogidos, tanto del alumnado como de las familias, en un municipio tipo (Herrera, provincia de Sevilla), se concluyó que el estado el grado general de CA de la población en conjunto es aceptable, con un valor general encima del valor medio. Las puntuaciones son algo menores en el grupo de las familias, y ligeramente mayor en el del alumnado. Comparando ambos colectivos, en la dimensión cognitiva es en la que se pueden detectar mayores diferencias entre los dos grupos de estudio. También en la dimensión activa encontramos diferencias en la puntuación del alumnado y las familias, siendo la de este último grupo considerablemente mayor. Por lo que podemos decir que las conductas proambientales parecen estar mucho más interiorizadas en el día a día de la población adulta, siempre que sean actividades de bajo coste, como el reciclaje, pero no necesariamente en actividades más costosas e inconvenientes como evitar el uso del vehículo privado (Moissander, 1998, cit. por Kollmuss y Agyeman, 2002).

En relación al objetivo de establecer la relación que pueda existir entre la CA del alumnado y la de sus familias podemos señalar que a priori no se observan tantas diferencias entre la CA del alumnado y la de las familias como cabría esperar, ni podemos decir que un grupo tiene un mayor nivel de CA que el otro (ambos grupos presentan promedios totales muy similares), lo que ha imposibilitado establecer sus niveles de interdependencia.

Más recientemente, a través de nuevas investigaciones se han explorado algunas variables más a fin de ir determinando aquellas que pudieran ser más relevantes en la búsqueda emprendida:

Así, por ejemplo, en el Trabajo Fin de Máster Dimensión afectiva y género Análisis de las creencias ambientales del alumnado de $5^{\circ}$ y $6^{\circ}$ de Educación Primaria (Heredia, 2018), se observa que el alumnado presenta una perspectiva pesimista en relación con la dimensión "gravedad de la problemática ambiental", lo que se traduce en un alto grado de emociones y sentimientos de preocupación por la misma. Se constata además que son minoritarias las perspectivas optimistas que restan gravedad o importancia a los problemas ambientales.

Otro de los objetivos específicos planteados en esta investigación era profundizar sobre el concepto denominado hipermetropía ambiental, definida como la tendencia a presentar una mayor preocupación por una problemática ambiental cuanto más alejada esté $\mathrm{y}$, además, mostrar un menor sentimiento de responsabilidad para solventar el problema (Uzzell, 2000). Se pudo comprobar que los resultados obtenidos confirman esa tendencia en el alumnado, al considerar por ejemplo más importantes los problemas a nivel mundial que los problemas locales. 
Por último, se analizó si el género condiciona la percepción del alumnado hacia el medio ambiente, confirmando que, en este caso, el género no condiciona dichas creencias o percepciones.

Igualmente se ha abordado un estudio de comparación de la CA entre el alumnado de dos colegios separados tanto geográficamente como de entorno socioeconómico: Incidencia del entorno económico y social en la definición de la CA del alumnado (Leal, 2018), comparando la tipificación de la CA entre dos poblaciones de alumnado de dos centros de Educación Primaria con circunstancias socioeconómicas y culturales muy distantes.

Según los resultados obtenidos, la profesión de los padres no parece tener relevancia en la conformación de la CA del alumnado. Tampoco se encontraron diferencias significativas entre alumnado de los diferentes contextos. Solo se aparecen algunas diferencias al analizar las diferentes dimensiones de la CA al confrontar el ámbito cognitivo, aunque es razonable pensar que son diferencias meramente contextuales referidas más al interés diferencial de diferentes temas que a variables realmente significativas para la CA.

\section{Conclusiones sobre la formación de la conciencia ambiental}

Analizados en conjunto los diferentes esfuerzos de investigación realizados, estamos en condiciones de dibujar una propuesta de modelo de formación de la CA de la población juvenil en nuestro contexto social y geográfico.

Por una parte, comenzamos estos esfuerzos después de determinar que la CA del alumnado de la universidad viene previamente determinada a su ingreso en la universidad. De tal forma esto es así, que esta no varía significativamente a lo largo de sus estudios universitarios y, es más, la elección de estos estudios parece estar determinada en gran medida por la CA previa del alumnado (Gomera, Villamandos y Vaquero, 2012) y que la categorización de esta conciencia ambiental nos dibujaba una población de la Universidad de Córdoba dividida en entres grandes grupos: aquellos con una CA mayor, los de una CA menor y sujetos no condicionados por la CA (Gomera, Villamandos y Vaquero, 2012).

Paralelamente, llegamos a la publicación de la metodología empleada para obtener de forma sencilla los indicadores de creencias ambientales utilizando la escala NEP modificada (Gomera, Villamandos y Vaquero, 2013).

A partir de aquí, iniciamos varias investigaciones, en el seno del Máster Interuniversitario de Educación Ambiental, la primera de las cuales nos rindió como resultado principal la confirmación de que existe una correlación entre los niveles de CA que trae el alumnado de nuevo ingreso en la universidad y el centro de procedencia (Gil, 2016).

Quedaba, por tanto, identificar variables más concretas que podrían determinar esta conformación diferencial de la CA del alumnado en su centro preuniversitario. Así se realizó un estudio que vino a confirmar que la CA del alumnado se ve fortalecida cuando el centro docente se involucra decididamente en un proyecto como Ecoescuelas (Morales, 2016).

Abordamos también algunas otras variables que, al menos a priori, podrían parecer sensibles a la formación de esa CA del alumnado. Así, en el estudio de Águila (2017) 
se analizó la CA del alumnado en comparación con los miembros parentales de sus familias. Los resultados obtenidos no nos permitieron establecer correlaciones positivas entre la CA de los padres y sus hijos.

Tampoco resultaron significativas otras variables analizadas en estudios posteriores (Heredia, 2018) como la de género cuando analizamos la dimensión afectiva, aunque sí que encontramos una prevalencia del concepto de hipermetropía ambiental en la población analizada de alumnado de primaria.

Finalmente, también hemos realizado el intento de comparar entornos económicos y sociales y encontrar en ellos diferencias entre la CA del alumnado. En el estudio realizado por Leal (2018) comparando los resultados de la CA entre dos colegios de entornos socioeconómicos muy dispares, tampoco se encontraron diferencias significativas ni por la profesión de los padres, ni por el tipo de colegio (rural y urbano) ni por la procedencia del alumnado (más nacional en un caso y de población emigrante otro). Las diferencias encontradas tenían más que ver con la dimensión cognitiva, que supone un interés diferencial en temas concretos, pero no una verdadera diferencia de los niveles de CA.

En definitiva, esta línea versa sobre el diseño y aplicación en distintos contextos de un instrumento para la medición y categorización de la CA en el alumnado, que indaga además en las causas de esta distribución, para comprobar si la Universidad está contribuyendo a su construcción y fortalecimiento. El modelo puede ser tenido en cuenta para el planteamiento de estrategias de educación y gestión ambiental, así como instrumento de evaluación de los procesos de sostenibilización curricular en las universidades, siendo de utilidad para abrir camino en la toma de decisiones sobre política ambiental y curricular de la institución educativa. Sobre este ámbito versa la segunda línea principal de investigación de nuestro equipo, que exponemos a continuación.

\section{Sobre el proceso de sostenibilización curricular y el profesorado como catalizador}

Como ya se ha comentado, debemos ser conscientes del papel de la educación superior para contribuir al desarrollo sostenible y a la mejora del conjunto de la sociedad. Este rol fundamental ha quedado reflejado desde hace décadas en declaraciones que numerosas universidades han firmado, como la Declaración de Talloires (ULSF, 1990), la Carta Copernicus (CRE, 1994) o la más reciente Declaración de Aichi-Nagoya (UNESCO, 2014), que las comprometen a introducir el Desarrollo Sostenible en la formación que ofrecen.

Uno de los procesos clave para que las universidades puedan dar respuesta a los retos planteados es el denominado como ambientalización o sostenibilización curricular. Un grupo de trabajo interuniversitario creado en torno a este concepto en el seno de la Conferencia de Rectores de las Universidades Españolas (CRUE), en el que participan, entre otros, los autores del presente artículo, define la sostenibilización curricular como el proceso de incorporación de criterios de sostenibilidad en la enseñanza y el aprendizaje del alumno, de modo que la sostenibilidad impregne todas las esferas de la docencia y, por ende, de la gestión en la que se desenvuelve la acción (Grupo de Sostenibilización Curricular de la CRUE, 2009). La ambientalización o 
sostenibilización curricular es Educación Ambiental realizada en la universidad (Capdevila, 1999). Hablamos, tal y como escribe Geli (2002), de:

Un proceso continuo de producción cultural tendente a la formación de profesionales comprometidos con la búsqueda permanente de las mejores relaciones posibles entre la sociedad y la naturaleza, atendiendo a los valores de la justicia, la solidaridad y la equidad, aplicando los principios éticos universalmente reconocidos y el respeto a las diversidades (p. 11).

Así pues, cabe preguntarse si la transformación real de las instituciones universitarias y sus prácticas están desarrollándose al mismo nivel y de forma coherente en relación al papel tan importante que las declaraciones y acuerdos institucionales y los expertos en la materia otorgan a la Universidad como avanzadilla en la senda de la sostenibilidad (Gutiérrez, Benayas y Calvo, 2006; Martínez-Agut et al. 2007).

Parecía necesario, por tanto, profundizar en la problemática que señalaban los profesionales e investigadores dedicados a la sostenibilización curricular. Era importante clarificar la situación del proceso de sostenibilización curricular en la educación superior española con objeto de detectar los aspectos clave en la evolución del mismo y orientar trabajos posteriores sobre la priorización de estrategias para avanzar en este camino. Ello además podía servir para delimitar el papel de los distintos agentes implicados, en particular del profesorado por el relevante potencial catalizador que podía tener, e indicar qué teclas se deberían tocar para acelerar el proceso de introducción de la sostenibilidad en la docencia universitaria.

Por todo ello, se desarrolló una investigación al respecto que quedó reflejada en una tesis doctoral (Antúnez, 2017) y dio lugar a un artículo (Antúnez, Gomera y Villamandos, 2017) y a distintas comunicaciones en congresos nacionales e internacionales (por ejemplo Antúnez y Gomera, 2013; Antúnez, Gomera y Villamandos, 2015; Antúnez, Gomera y Villamandos, 2016). Dicha investigación quería intentar dar respuesta a preguntas como: ¿Cuáles son los principales problemas del proceso de sostenibilización curricular en España? ¿Cómo influyen esos problemas en el estado actual de este proceso? ¿Qué catalizadores se pueden poner en marcha para dar pasos en las vías de solución de estos problemas?

Así, la primera fase de la investigación fue un análisis del estado del proceso de sostenibilización curricular en las universidades españolas. Para ello se identificaron los principales problemas en este ámbito, se ordenaron por su nivel de influencia en el estado del proceso de sostenibilización curricular en España y se exploraron posibles soluciones. Como unidad de análisis se utilizó al Grupo de Sostenibilización Curricular de la Comisión CRUE-Sostenibilidad de la CRUE, cuyos miembros fueron considerados como expertos y expertas en este campo, a los que se les pasó una plantilla estandarizada con una serie de preguntas en dos tandas.

Los problemas detectados, ordenados por la influencia que tienen en el proceso de sostenibilización curricular en España según los expertos consultados, fueron los siguientes (figura 1) (Antúnez, 2017):

\section{Existe poca formación en sostenibilidad para profesorado universitario,} poco estructurada y de carácter voluntario.

2. Existe entre la comunidad universitaria un escaso conocimiento de los conceptos de sostenibilidad y sostenibilización curricular. 
3. Hay un compromiso institucional con la sostenibilidad limitado y lejos de una política activa por una sostenibilización integral de las universidades.

4. Hay dificultades para llevar a la práctica la teoría sobre la inclusión de la sostenibilidad en el diseño curricular, en contenidos, metodologías, valores y evaluación.

5. Hay escasa investigación en sostenibilización curricular y está poco valorada y poco reconocida. (p. 70)

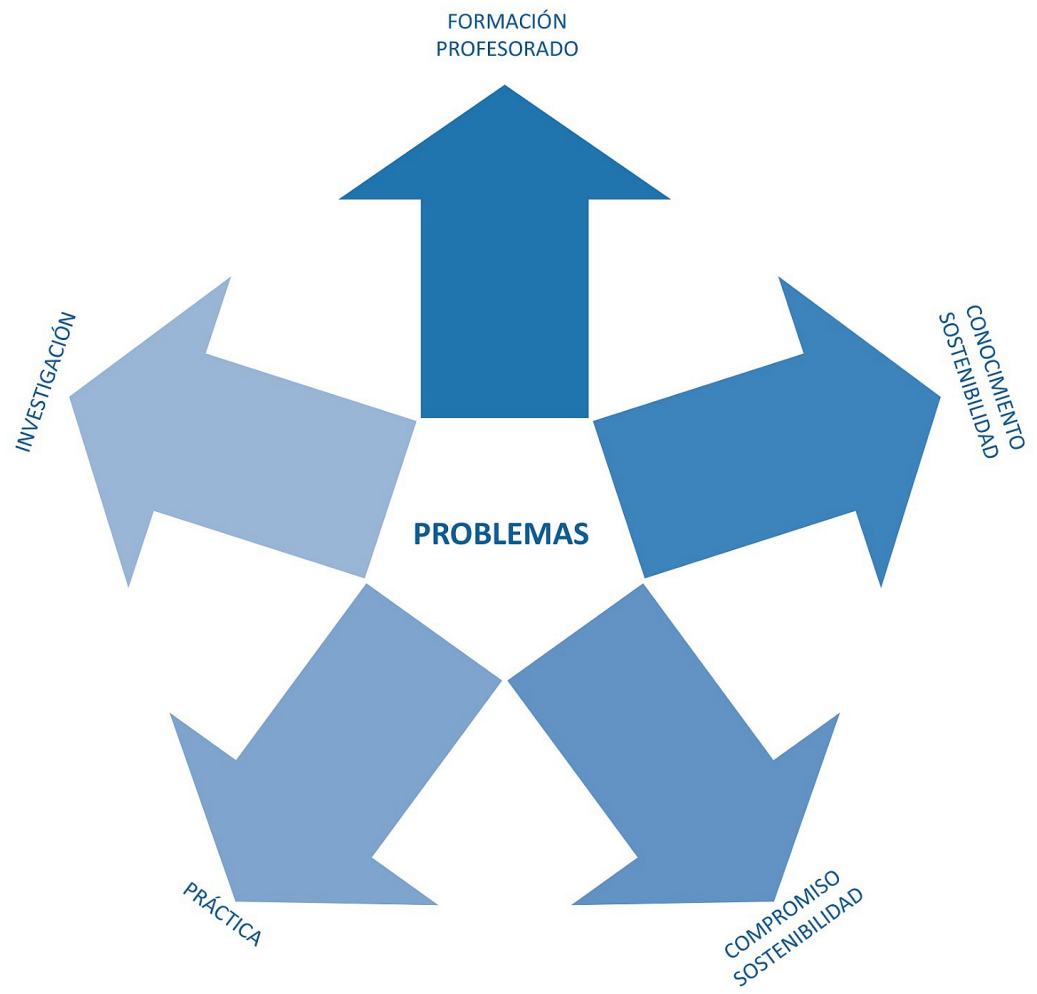

Figura 1. Categorización de los problemas detectados en el proceso de sostenibilización curricular en las universidades españolas (Antúnez, 2017; p. 68).

Las soluciones aportadas por los expertos consultados por cada problema fueron identificadas y categorizadas hasta alcanzar un listado que no reproduciremos aquí por falta de espacio, pero que puede consultarse al completo en Antúnez (2017). Puede verse un resumen en la Figura 2.

Los problemas señalados por los expertos consultados son coherentes con otros estudios previos, tanto a nivel nacional como internacional (Orr, 1992; Gutiérrez, Benayas y Calvo, 2006; Martínez-Agut et al. 2007; Azcárate, Navarrete y García, 2012; Banga Chhokar, 2012; Ferreira y Tilbury, 2012; Vilches y Gil-Pérez, 2012; Aznar Minguet, Ull, Piñero y Martínez-Agut, 2014; Mulà, Tilbury y Ryan, 2015). Dichos problemas conforman un sistema complejo y, como en cualquier sistema de este tipo, hay múltiples variables que considerar, todas necesarias, a la hora de transformarlo.

Pero los resultados de nuestro trabajo indican que el papel del profesorado y de su actividad docente es esencial. Es el profesorado quien imparte las asignaturas y forma a los futuros profesionales, quien planifica las actividades de formación para el profesorado, quien prioriza unos temas de investigación sobre otros, quien termina desempeñando puestos de responsabilidad en departamentos, decanatos, consejos de gobierno de universidades o comisiones académicas de distinto tipo (Antúnez, 2017). 
El profesorado, por tanto, es el actor clave para alcanzar de forma eficiente una transformación global en los procesos de enseñanza aprendizaje (Vilches y Gil-Pérez, 2012) en las universidades que los orienten en el mismo sentido y dirección que los compromisos por la sostenibilidad.

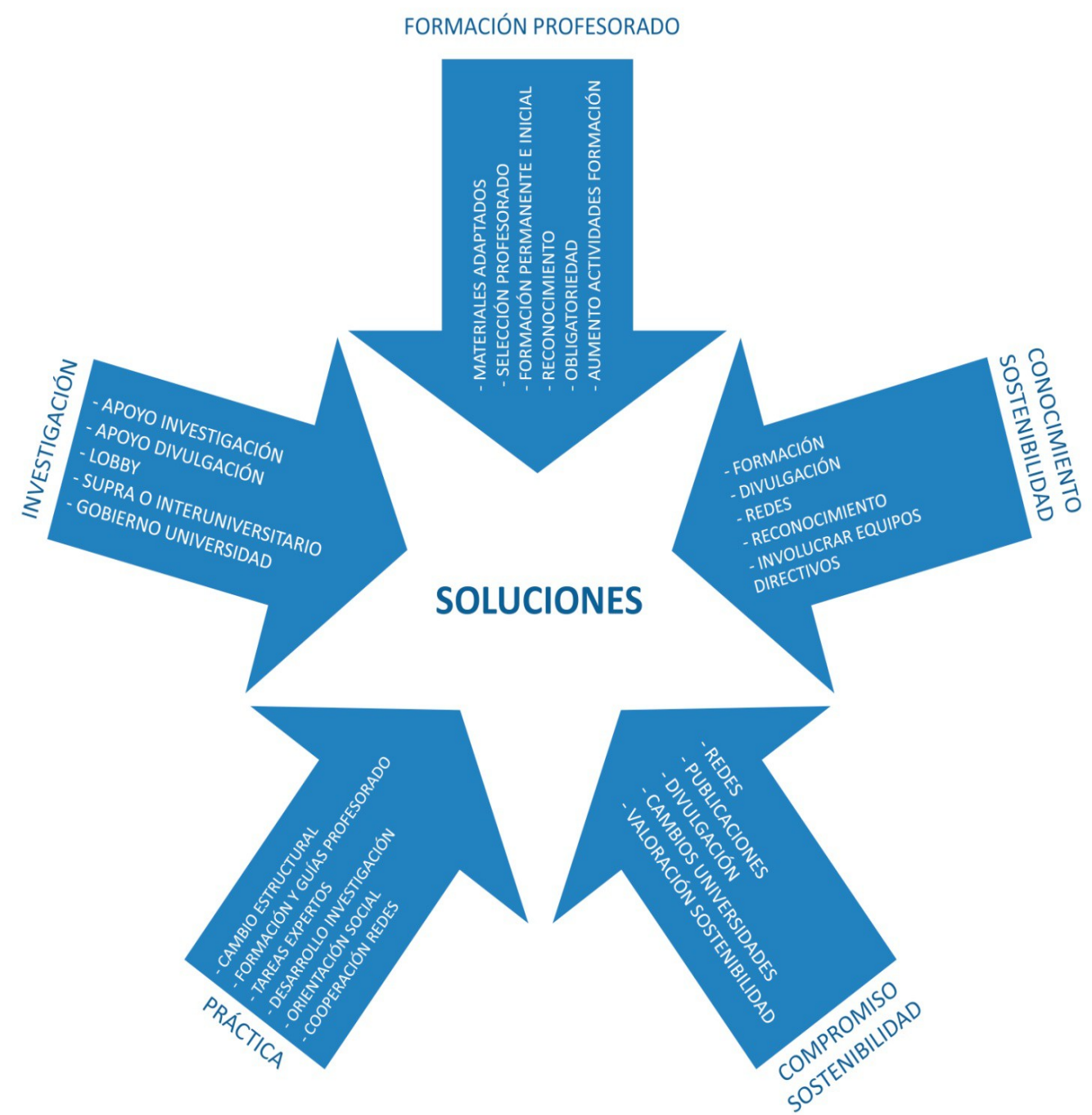

Figura 2. Resumen de las soluciones aportadas por los expertos para mejorar el proceso de sostenibilización curricular en las universidades españolas (Antúnez, 2017; p. 71).

Por ello, parece coherente pensar que las acciones dirigidas al profesorado pueden ser las más eficientes para provocar una transición más rápida. Cada docente tiene el potencial de convertirse en un catalizador para introducir de forma efectiva y lo más rápidamente posible la sostenibilidad en la docencia universitaria (Antúnez, 2017).

Por tanto, aunque la situación de la sostenibilización curricular en España responde a múltiples factores, es coherente que los resultados de nuestra investigación destaquen el papel del profesorado y su escasa formación en sostenibilidad y sostenibilización curricular como el problema con más incidencia en este proceso. Además, como deja patente la opinión de los expertos, la formación integral para el profesorado ofrecida por las universidades a este respecto está aún por desarrollarse al nivel que parece necesario. 
Es por esto que creemos que los principales esfuerzos para acelerar el proceso de sostenibilización curricular de nuestras universidades deberían volcarse en el profesorado. Su formación, tanto en sostenibilidad como en la manera de llevar a cabo la sostenibilización en la docencia, es algo que, aunque se empieza a extender (Banga Chhokar, 2012; Ferreira y Tilbury, 2012; Vilches, Macías y Gil-Pérez, 2014), tiene aún mucho que avanzar para implantarse de forma mayoritaria.

\section{La formación del profesorado como catalizador de la sostenibilización de la Universidad}

Al hilo de lo anterior, desde el equipo de investigación nos planteamos las siguientes preguntas: ¿Es posible poner en marcha procesos eficaces de formación del profesorado en sostenibilización curricular sin esperar a grandes cambios institucionales? ¿Con qué herramientas cuenta el profesorado para trabajar la sostenibilización curricular? ¿Cómo se puede mejorar la eficacia de la introducción de competencias de sostenibilidad en la docencia universitaria?

Para tratar de avanzar en su respuesta, encontramos un escenario muy propicio, ya que precisamente el equipo autor de este trabajo forma parte del grupo que coordina e imparte el curso de formación de profesorado universitario Sostenibilización curricular: Introducción de la sostenibilidad en la docencia universitaria en la Universidad de Córdoba, el cual lleva celebrándose desde el curso 2007-08, con una media de 30 participantes por edición y alcanzando en el curso 2018-19 su décima. Constituye un contexto excelente para poner en práctica las sinergias mencionadas entre los perfiles técnico, de gestión, docente e investigador de nuestro equipo.

Siguiendo las directrices aprobadas por la Conferencia de Rectores de las Universidades Españolas (CRUE), este curso busca reflexionar sobre la responsabilidad del profesorado universitario en la incorporación de criterios de sostenibilidad en su actividad docente, así como de transmitir las herramientas puestas en práctica en las universidades en los procesos de sostenibilización curricular a la hora de diseñar, impartir y evaluar las asignaturas.

De los contenidos, resultados, propuestas y metodologías desarrollados en el curso han surgido interesantes contribuciones científicas, como la experiencia en su diseño, desarrollo y evaluación (Gomera, Antúnez, Villamandos y Vaquero, 2015), la exploración de un taller práctico que se realiza sobre identificación y evaluación de competencias en sostenibilidad en los estudios universitarios (Antúnez, Gomera, De Toro, Vaquero y Villamandos, 2011), el análisis de los ejercicios de los participantes del curso para evaluar cómo se enfrenta el profesorado a su guía docente en materia de sostenibilidad (Antúnez, Gomera, Villamandos, De Toro y Vaquero, 2017), o el estudio de la percepción del profesorado sobre la Agenda 2030 en el marco del curso y de la red de colaboración e intercambio de experiencias que ha surgido a raíz del mismo (Antúnez, Gomera, Vaquero y Villamandos, 2018).

Incluso se ha realizado un Trabajo de Fin del Máster Interuniversitario de Educación Ambiental (De Paula, 2016), que analiza el impacto de este curso de formación sobre el profesorado universitario que ha participado en el mismo en las distintas ediciones tanto en el ámbito personal así como en situaciones de aula.

Tras las diez ediciones celebradas, el curso permite adquirir los siguientes resultados de aprendizaje (Tabla 1): 
Tabla 1. Resultados de aprendizaje del curso Sostenibilización curricular: Introducción de la sostenibilidad en la docencia universitaria de la Universidad de Córdoba.

1. Las consecuencias de la actual relación del ser humano con el medio ambiente está visibilizando una crisis sistémica sin precedentes. Este desajuste global hace necesario educar ambientalmente a todos los niveles.

2. Existe la necesidad de transformar la educación superior para abordar los retos de la crisis global en el marco de la sostenibilidad.

3. Las universidades tienen un papel fundamental para lograr el cumplimiento de los Objetivos de Desarrollo Sostenible (ODS), a la vez que pueden beneficiarse enormemente al comprometerse con la Agenda 2030.

4. Uno de los mayores retos de las universidades es formar profesionales críticos con el curso actual de nuestra sociedad y capaces de actuar para promover un desarrollo más sostenible.

5. El profesorado universitario tiene responsabilidad y oportunidad para contribuir a resolver la crisis ambiental actual mediante la incorporación de criterios de sostenibilidad en la enseñanza y el aprendizaje del alumnado, de modo que la sostenibilidad impregne todas las esferas de la docencia.

6. La sostenibilización curricular va más allá de lo ambiental y más allá de los conocimientos de las asignaturas. Hay que hacer cambios en todo el proceso de enseñanza-aprendizaje: en competencias, metodología, contenidos, evaluación y buenas prácticas.

7. Cualquier titulación contempla o puede contemplar competencias relacionadas con sostenibilidad, de manera directa o indirecta.

8. Cualquier futuro profesional de cualquier área puede desarrollar su labor desde la perspectiva de la sostenibilidad.

9. Es posible trabajar con el profesorado, en su formación y en sus competencias, sin esperar a cambios más profundos en la planificación académica o en las propias instituciones universitarias.

10. No hay fórmulas ni recetas mágicas, puesto que hay aún mucho que recorrer y muchas cuestiones sin resolver. Por suerte ya existen investigaciones, herramientas, redes y experiencias a disposición de las universidades para avanzar en la sostenibilización curricular.

Tras estas ideas fuerza subyace gran parte del sentido del trabajo de investigación en Educación Ambiental desarrollado por nuestro equipo, entendiéndola como una herramienta práctica y determinante para la resolución de los problemas ambientales, y con el contexto de la Universidad como escenario fundamental para el impulso de nuevas vías hacia la sostenibilidad.

\section{Agradecimientos}

Los autores de este trabajo queremos agradecer al profesorado y alumnado del Máster Interuniversitario de Educación Ambiental su colaboración y contribuciones en las investigaciones desarrolladas en el campo objeto del artículo. También a la Junta de Andalucía por su apoyo en la financiación de actividades del Aula de Sostenibilidad, y por supuesto a la comunidad universitaria de la UCO y su Equipo de Gobierno, por su compromiso por una universidad más sostenible.

\section{Referencias}

Águila, L. (2017). Análisis comparado de la conciencia ambiental en educación secundaria: la visión del alumnado y sus familias. Trabajo Fin de Máster Interuniversitario en Educación Ambiental, sin publicar defendido en la Universidad de Córdoba.

Amérigo, M., Aragonés, J. I., Sevillano, V. y Cortés, B. (2005). La estructura de las creencias sobre la problemática medioambiental. Psicothema, 17(2), 257-262. Disponible en: http://www.psicothema.com/pdf/3096.pdf 
Antúnez, M. (2017). La problemática del proceso de sostenibilización curricular en el contexto universitario español: La formación del profesorado como catalizador. Tesis doctoral por la Universidad de Córdoba. https://helvia.uco.es/xmlui/handle/10396/15069

Antúnez, M. y Gomera, A. (2013). Sostenibilidad y currículum: una foto del pasado y el presente para reflexionar sobre el futuro. Comunicación en el I Encuentro Universitario de Sostenibilización Curricular. Universidad Europea de Madrid. Disponible en http://hdl.handle.net/11268/6478

Antúnez, M., Gomera, A. y Villamandos, F. (2015). Sostenibilidad y currículum: problemática y posibles soluciones en el contexto universitario español. En Huertas, C., Serrano, R. y Gómez, M.E. (coords.), Libro de Resúmenes del I Congreso Internacional Educación y Cooperación al Desarrollo. Córdoba: Ediciones Don Folio. http://www.uco.es/educacionycooperacion/docs/Libro_de_abstracts.pdf

Antúnez, M., Gomera, A. y Villamandos, F. (2016). Un acercamiento a la problemática de la sostenibilización curricular universitaria en España. En T. RamiroSánchez, y Ramiro, M. T. (coords.), Avances en ciencias de la educación y del desarrollo, 2016. (pp. 43-49). Granada: Asociación Española de Psicología Conductual (AEPC).

Antúnez M., Gomera A. y Villamandos F. (2017). Sostenibilidad y Currículum: Problemática y posibles soluciones en el contexto universitario español. Profesorado. Revista de Currículum y Formación de Profesorado, 21(4), 197-214.

Antúnez, M., Gomera, A., Vaquero, M. y Villamandos, F. (2018). Percepción del profesorado universitario sobre la Agenda 2030: el caso de la Universidad de Córdoba. Libro de resúmenes del IV Congreso Internacional de Estudios del Desarrollo (p. 108). Córdoba, España: Universidad Loyola. Disponible en: https://drive.google.com/file/d/1VZYEerNWSQGJY-ocpiBdJJcYe-AlLG82/view

Antúnez, M., Gomera, A., De Toro, A., Vaquero, M. y Villamandos, F. (2011). Taller práctico: identificación y evaluación de competencias en sostenibilidad en los estudios universitarios. Comunicación presentada en el Encuentro Global University Network for Innovation: Universidades en Transición, Transformaciones para la Sostenibilidad. Barcelona.

Antúnez, M., Gomera, A., Villamandos, F., De Toro, A. y Vaquero, M. (2017). ¿Cómo se enfrenta el profesorado a su guía docente? Análisis de los ejercicios de los participantes en un curso de profesorado sobre sostenibilización curricular. En Lugo, M. y Limón-Domínguez, D. (coords.), X Seminario de investigaciones en educación ambiental y educación para el desarrollo sostenible: nuevos escenarios, retos y propuestas para el reequilibrio sustentable (pp. 43-48). Madrid: Ministerio de Medio Ambiente.

Aula de Sostenibilidad (2017). Reglamento del Aula de Sostenibilidad de la Universidad de Córdoba. Recuperado de http://www.uco.es/catedrasyaulas/aulasostenibilidad/Enlaces_y_descargas/r eglamento_AS.pdf 
Azcárate, P., Navarrete, A. y García, E. (2012). Aproximación al nivel de inclusión de la sostenibilidad en los curricula universitarios. Profesorado: Revista de currículum y formación del profesorado, 16(2), 105-119.

Aznar Minguet, P., Ull, M.A., Piñero, A. y Martínez-Agut, M.P. (2014). La sostenibilidad en la formación universitaria: Desafíos y oportunidades. Educación XX1, 17(1), 133-158. doi: 10.5944/educxx1.17.1.10708

Banga Chhokar, K. (2012). Education for sustainable development in higher education in India. Global University Network for Innovation (GUNI), Higher education in the world, 4. Higher Education's Commitment to Sustainability: from Understanding to Action, (pp. 99-102). Barcelona, España: GUNI.

Benayas, J. y Alba, D. (2011). Evolución y tendencias de la incorporación de la sostenibilidad en las universidades españolas. En P. Silva, A. Pasevi, A. Alba y M. J. Díaz (coords.), Visiones y Experiencias Iberoamericanas de Sostenibilidad en las Universidades, (pp. 29-35). Sâo Paulo, Brasil: Universidade de Sâo Paulo.

Benayas, J., Alba, D. y Sánchez, S. (2002). La ambientalización de los campus universitarios: El caso de la Universidad Autónoma de Madrid. Revista Ecosistemas, 11(3), 1-8.

Breiting, S. (1997). Hacia un nuevo concepto de educación ambiental. Carpeta Informativa del CENEAM. Recuperado de https://www.miteco.gob.es/ca/ceneam/articulos-de-opinion/1997sorenbreiting_tcm34-163533.pdf

Capdevila, I. (1999). L'ambientalització de la universitat. Col·lecció de Monografies d’Educació Ambiental (no 6). Palma de Mallorca: di7Edició.

Chuliá, E. (1995). La Conciencia Ambiental de los Españoles en los Noventa. ASP Research Papers, 12, 1-32.

CRE (1994). Carta Copernicus: The University Charter for Sustainable Development. https://www.iau-hesd.net/sites/default/files/documents/copernicus.pdf

De Paula, F. (2016). Análisis del impacto de la sostenibilización curricular a través del curso de formación "Sostenibilidad y medio ambiente en la docencia" de la Universidad de Córdoba. Trabajo Fin de Máster Interuniversitario en Educación Ambiental sin publicar defendido en la Universidad de Córdoba.

Dunlap, R. E. y Van Liere, K. D. (1978). The new environmental paradigm. Journal of Environmental Education, 9, 10-19.

Dunlap, R. E., Van Liere, K. D., Merting, A. G. y Jones, R. E. (2000). Measuring endorsement of the New Ecological Paradigm: A revised NEP scale. Journal of Social Issues, 56(3), 425-442.

Febles, M. (2004). Sobre la necesidad de la formación de una conciencia ambiental [About the necessi-ty of constructing environmental awareness]. La Habana, Cuba: Universidad de La Habana

Ferreira, J. y Tilbury, D. (2012). Higher education and sustainability in Australia: transforming experiences. Global University Network for Innovation (GUNI), Higher education in the world, 4. Higher Education's Commitment to 
Sustainability: from Understanding to Action, (pp. 96-99). Barcelona, España: GUNI.

Geli, A. (2002). Introducción. Universidad, Sostenibilidad y Ambientalización Curricular. En M. G. Junyent, M. Junyent, A. Geli, y E. Arbat (eds.). Ambientalización curricular de los estudios superiores. Tomo I (pp. 11-18). Girona: Universitat de Girona. Servicio de Publicaciones.

Gil, I. (2016). Análisis y medición de la conciencia ambiental en el alumnado de primer curso de la Universidad de Córdoba. Trabajo Fin de Máster Interuniversitario en Educación Ambiental sin publicar defendido en la Universidad de Córdoba.

Gomera, A. (2011). Análisis, medición y distribución de la conciencia ambiental en el alumnado universitario: una herramienta para la educación ambiental. Tesis Doctoral sin publicar. Universidad de Córdoba, Córdoba.

Gomera, A., Villamandos, F. y Vaquero, M. (2012) Medición y categorización de la conciencia ambiental del alumnado universitario: contribución de la universidad a su fortalecimiento. Profesorado. Revista de Currículum y Formación de Profesorado, 16(2). 193-212.

Gomera, A., Villamandos, F. y Vaquero, M. (2013) Construcción de indicadores de creencias ambientales a partir de la escala NEP. Acción Psicológica, 10(1). 149153

Gomera, A., Antúnez, M., Villamandos, F., y Vaquero, M. (2015). La formación del profesorado universitario en sostenibilidad y medio ambiente: Experiencia en el diseño y desarrollo de cursos específicos. En C. Huertas, R. Serrano y M. E. Gómez (coords.), Libro de Resúmenes del I Congreso Internacional Educación y Cooperación al Desarrollo. Córdoba: Ediciones Don Folio. Disponible en http://www.uco.es/educacionycooperacion/docs/Libro_de_abstracts.pdf

Grupo de Sostenibilización Curricular de la CRUE (2009). Página web del Grupo de Trabajo de Sostenibilización curricular de la Comisión CRUE-Sostenibilidad. Recuperado de https://sostecurricularcrue.wordpress.com/about/

Gutiérrez J., Benayas, J. y Calvo, S. (2006). Educación para el desarrollo sostenible: Evaluación de retos y oportunidades del decenio 2005-2014. Revista Iberoamericana de Educación, 40, 25-69.

Heredia, M. (2018). Dimensión afectiva y género Análisis de las creencias ambientales del alumnado de $5^{o}$ y $6^{o}$ de Educación Primaria. Trabajo Fin de Máster Interuniversitario en Educación Ambiental sin publicar defendido en la Universidad de Córdoba.

Jiménez, M. y Lafuente, R. (2006). La operacionalización del concepto de conciencia ambiental en las encuestas. En R. de Castro (coord.), Persona, Sociedad y Medio Ambiente (pp. 121-150). Sevilla: Junta de Andalucía.

Junta de Andalucía (2006). Estrategia Andaluza de Educación Ambiental. Recuperado de https://www.uco.es/catedrasyaulas/aulasostenibilidad/descargas/eadea.pdf

Junta de Andalucía (2015). Dossier Informativo Programa "Red Andaluza de Ecoescuelas 2015/2016". Recuperado de 
http://www.juntadeandalucia.es/educacion/webportal/ishareservlet/content/dfae988f-5367-4e8f-bcf6-c7fcbadb10ac

Kollmuss, A. y Agyeman, J. (2002). Mind the gap: Why do people act environmentally and what are the barriers to pro-environmental behavior? Environmental Education Research 8(3), 239-260.

Leal, M. (2018) Incidencia del entorno económico y social en la definición de la CA del alumnado. Trabajo Fin de Máster Interuniversitario en Educación Ambiental sin publicar defendido en la Universidad de Córdoba.

León-Fernández, Y., Gomera, A., Antúnez, M., Martínez-Escrich, B., Villamandos, F y Vaquero, M. (2017) Enhancing environmental management in universities through participation: the case of the University of Córdoba, Journal of Cleaner Production, 172, 4328-4337. https://doi.org/10.1016/j.jclepro.2017.06.103

Martínez-Agut, M. P., Aznar, P., Ull, M. Á., y Piñero, A. (2007). Promoción de la sostenibilidad en los curricula de la enseñanza superior desde el punto de vista del profesorado: un modelo de formación por competencias. Educatio Siglo XXI, 25, 187-208.

Ministerio de Medio Ambiente (1999). Libro Blanco de la Educación Ambiental. Madrid: Ministerio de Medio Ambiente.

Morales, E. (2016). Incidencia del Programa Ecoescuela en la definición de la conciencia ambiental del alumnado. un estudio comparado. Trabajo Fin de Máster Interuniversitario en Educación Ambiental. Disponible en https://helvia.uco.es/xmlui/bitstream/handle/10396/15893/TFM_Elizabeth_ Morales_Rojas.pdf

Mulà, I., Tilbury, D. y Ryan, A. (2015). Mapping opportunities for developing Education for Sustainable Development (ESD) competences. University of Gloucestershire, United Kingdom: US4SD North - Region. DOI:10.13140/RG.2.1.1773.8966.

Orr, D. (1992). Ecological literacy: Education and the transition to a postmodern world. Albany: State University of New York.

Ruiz, J. (2006). Mentalidades medioambientales: los discursos sobre el medio ambiente de los andaluces residentes en zonas urbanas. Papers. Revista de sociología, $81,63-88$.

Sauvé, L. (2005). Uma cartografia das corrientes em educaçao ambiental. In M. Sato, et I. Carvalho (Dir.). Educação ambiental -Pesquisa e desafios. (pp. 17-46). Porto Alegre: Artmed. (En castellano, Cátedra de investigación de Canadá en educación ambiental. Université du Québec à Montréal) recuperado de http://www.ecominga.uqam.ca/PDF/BIBLIOGRAPHIE/GUIDE_LECTURE_3/1/ 2.Sauve.pdf

Tilbury, D. (2012) Higher education for sustainability: a global overview of commitment and progress. Higher education in the world, 4, Higher Education's Commitment to Sustainability: from Understanding to Action, 18-28.

ULSF (1990). Declaración de líderes de universidades para un futuro sostenible. Declaración de Talloires. 
UNESCO (2014). Aichi-Nagoya Declaration on Higher Education for Sustainable Development. Recuperado de http://www.unesco.org/new/fileadmin/MULTIMEDIA/HQ/ERI/pdf/AichiNagoya_Declaration_EN.pdf

Uzzell, D. (2000).The psyco-spatial dimension of global environmental problems. Victoria (Canada): Journal of Environmental Psychology, 20(3), 307-318.

Valencia, A., Arias, M. y Vázquez, R. (2010). Opiniones y actitudes: ciudadanía y conciencia medioambiental en España. Madrid: Centro de Investigaciones Sociológicas.

Vilches, A. y Gil-Pérez, D. (2012). La educación para la sostenibilidad en la Universidad: el reto de la formación del profesorado. Profesorado. Revista de curriculum y formación del profesorado, 16(2), 25-43.

Vilches, A., Macías, O. y Gil-Pérez, D. (2014). La transición a la sostenibilidad: un desafío urgente para la ciencia, la educación y la acción ciudadana. Temas clave de reflexión y acción. Documentos de trabajo de Iberciencia, (01). Recuperado de: http://www.ibercienciaoei.org/documentoiberciencia1.pdf

Vozmediano, L. y San Juan, C. (2005). Escala Nuevo Paradigma Ecológico: propiedades psicométricas con una muestra española obtenida a través de Internet. Medio Ambiente y Comportamiento Humano, 6(1), 37-49. 